\title{
GW23-e2072 CLINICAL STUDY OF THE MECHANISM OF MITRAL REGURGITATION IN THE PAPILLARY MUSCLE DYSFUNCTION
}

doi:10.1136/heartjnl-2012-302920ad.27

${ }^{1}$ Su Maolong, ' Wang Jing, 'Zhang Nan, ${ }^{2}$ Su Maolong. ${ }^{1}$ Zhongshan Hospital Xiamen University; ${ }^{2}$ Echocardiography Laboratory, Zhongshan Hospital Xiamen University. Xiamen, Fujian, 361004, P. R. China (Tel \& Fax: 86-592-2212328)

Objectives 目的: This investigation and report was a clinical study of the mechanism of mitral regurgitation in the papillary muscle dysfunction caused by coronary artery disease. We have studied correlation of the different geometric angles between the mitral valve leaflets and the mitral valve annular and mitral regurgitation severity by using transesophageal echocardiography (TEE) .

Methods 104 cases patients, among 44 cases patients with mitral regurgitation and the papillary muscle dysfunction caused by posterior or inferior myocardial ischaemia or infarction by coronary artery disease, intraoperative TEE were recorded in 10 patients after complex mitral valve repair including placement of an annular ring with or without concurrent repair of mitral leaflet tissue itself, and 20 patients control subjects were studied. TEE showed below three view: left ventricular four-chamber view, two-chamber view and apical long-axis view during early systole and lately systole; the geometric angles between the mitral valve leaflets and mitral valve annular in three views were measured by SIEMENS KinetDx DS3000 software system. The geometric angles between the mitral valve anterior leaflet with the mitral valve annular was determined as $\mathrm{Aá}^{\circ}$, the geometric angles between the mitral valve posterior leaflet with the mitral valve annular was 
determined as $\mathrm{Pa}^{\circ}$, the geometric distance from closed point the anterior and posterior leaflets tip of the mitral valve to the mitral valve annular was determined as $\mathrm{d}(\mathrm{cm})$, early systole was determined as S1, lately systole was determined as S2, and the geometric maximum area from closed point the anterior with posterior leaflets tip of the mitral valve to the mitral valve annular was determined as Area-max $\left(\mathrm{cm}^{2}\right)$.

Results The geometric angles between the mitral valve leaflets and mitral valve annular in three planes of the group of the papillary muscle dysfunction caused by posterior or inferior myocardial ischaemia or infarction by coronary artery disease, both $\mathrm{Aá}^{\circ}, \mathrm{Pa}^{\circ}$, and $\mathrm{d}(\mathrm{cm})$ was significantly difference than the group of the control subjects during early systole and ately systole, respectively $(p<0.01)$. Among of all planes of three patients was showed $\mathrm{Pa}^{\circ}<\mathrm{Aa}^{\circ}$, but rest both $\mathrm{Pa}^{\circ}>\mathrm{Aa}^{\circ}$. Whereas the geometric angles between the mitral valve leaflets and mitral valve annular in three planes of the another group (20 cases patients of the papillary muscle dysfunction caused by posterior or inferior myocardial ischaemia or infarction by coronary artery disease) after complex mitral valve repair including placement of an annular ring with or without concurrent repair of mitral leaflet tissue itself was showed both Aá ${ }^{\text {, }}{ }^{\circ}$, and $\mathrm{d}(\mathrm{cm})$ no significant difference than control subjects during early systole and lately systole, respectively ( $p>0.05)$. Determination of the severity of the mitral regurgitation by proximal isovelocity surface area (PISA) by color Doppler flow imaging (CDFI). In the group of the papillary muscle dysfunction, effective regurgitant orifice (ERO, $\mathrm{cm}^{2}$ ) ranged from $0.20 \mathrm{~cm}^{2}$ to $0.67 \mathrm{~cm}^{2}\left(0.41 \pm 0.11 \mathrm{~cm}^{2}\right)$, which was significantly different from after the mitral valve surgical repair, where it ranged from 0 to $0.17 \mathrm{~cm}^{2}\left(0.06 \pm 0.04 \mathrm{~cm}^{2}, p<0.01\right)$; also, in the group of the papillary muscle dysfunction, Area-max $\left(\mathrm{cm}^{2}\right)$ ranged from $1.28 \mathrm{~cm}^{2}$ to $3.91 \mathrm{~cm}^{2}\left(2.20 \pm 0.77 \mathrm{~cm}^{2}\right)$, which was significantly different from after the mitral valve surgical repair, where it ranged from $0.46 \mathrm{~cm}^{2}$ to $1.75 \mathrm{~cm}^{2}(0.85$ $\pm 0.36 \mathrm{~cm}^{2}$, normal control subjects $\left.0.64 \pm 0.23 \mathrm{~cm}^{2} ; \mathrm{p}<0.001\right)$.

Conclusions The severity of mitral regurgitation with the papillary muscle dysfunction caused by coronary artery disease relies heavily on geometric maximum angles between mitral valve leaflets and mitral valve annular during early systole or lately systole ( $\max$ angles $\mathrm{r}=0.85$ ); likely it deformation from Páo $\max (r=0.79)$ with area-max $\left(\mathrm{cm}^{2}\right)(\mathrm{r}=0.69)$, but seem it no deformation from Aá $(\mathrm{r}=0.55)$, and $\mathrm{d}(\mathrm{cm}) \quad(\mathrm{r}=0.37)$. Therefore, TEE has been suggested as a helpful tool for differentiating the geometry angles between the mitral valve leaflets with the mitral valve annular planes and study of the mechanism of mitral regurgitation in the papillary muscle dysfunction 